\title{
Colonization With Extensively Drug-Resistant Acinetobacter baumannii and Prognosis in Critically III Patients: An Observational Cohort Study
}

OPEN ACCESS

Edited by:

Ana Afonso,

University of São Paulo, Brazil

Reviewed by:

Afsaneh Karmostaji,

Hormozgan University of Medical

Sciences, Iran

Maristela Freire,

São Paulo State University, Brazi

*Correspondence:

Hao Wang

wanghao34@126.com

Yuguo Chen

chen919085@sdu.edu.cn

†These authors have contributed equally to this work

Specialty section:

This article was submitted to Infectious Diseases - Surveillance,

Prevention and Treatment,

a section of the journal

Frontiers in Medicine

Received: 14 February 2021 Accepted: 06 April 2021

Published: 30 April 2021

Citation:

Zheng Y, Xu N, Pang J, Han H,

Yang $H$, Qin W, Zhang H, Li W,

Wang $H$ and Chen $Y$ (2021)

Colonization With Extensively

Drug-Resistant Acinetobacter baumannii and Prognosis in Critically III Patients: An Observational Cohort

Study. Front. Med. 8:667776. doi: 10.3389/fmed.2021.667776
Yue Zheng ${ }^{1,2,3,4 t}$, Nana X ${ }^{5 \dagger}$, Jiaojiao Pang ${ }^{1,2,3,4 \dagger}$, Hui Han ${ }^{6}$, Hongna Yang ${ }^{6}$, Weidong Qin ${ }^{6}$, Hui Zhang ${ }^{6}$, Wei $\mathrm{Li}^{7}$, Hao Wang ${ }^{1,6,8 *}$ and Yuguo Chen ${ }^{1,2,3,4 *}$

${ }^{1}$ Shandong Provincial Clinical Research Center for Emergency and Critical Care Medicine, Institute of Emergency and Critical Care Medicine of Shandong University, Chest Pain Center, Qilu Hospital of Shandong University, Jinan, China, ${ }^{2}$ Department of Emergency Medicine, Qilu Hospital of Shandong University, Jinan, China, ${ }^{3}$ Key Laboratory of Emergency and Critical Care Medicine of Shandong Province, Key Laboratory of Cardiopulmonary-Cerebral Resuscitation Research of Shandong

Province, Shandong Provincial Engineering Laboratory for Emergency and Critical Care Medicine, Qilu Hospital of Shandong University, Jinan, China, ${ }^{4}$ The Key Laboratory of Cardiovascular Remodeling and Function Research, The State and Shandong Province Joint Key Laboratory of Translational Cardiovascular Medicine, Chinese Ministry of Education, Chinese Ministry of Health and Chinese Academy of Medical Sciences, Qilu Hospital of Shandong University, Jinan, China,

${ }^{5}$ Cardiosurgery Care Unit, Department of Cardiosurgery, Qilu Hospital of Shandong University, Jinan, China, ${ }^{6}$ Department of Critical Care Medicine, Qilu Hospital of Shandong University, Jinan, China, ${ }^{7}$ Department of Clinical Laboratory, Qilu Hospital of Shandong University, Jinan, China, ${ }^{8}$ Department of Pharmacology, School of Basic Medical Sciences, Shandong University, Jinan, China

Background: Acinetobacter baumannii is one of the most frequently isolated opportunistic pathogens in intensive care units (ICUs). Extensively drug-resistant A. baumannii (XDR-AB) strains lack susceptibility to almost all antibiotics and pose a heavy burden on healthcare institutions. In this study, we evaluated the impact of XDR-AB colonization on both the short-term and long-term survival of critically ill patients.

Methods: We prospectively enrolled patients from two adult ICUs in Qilu Hospital of Shandong University from March 2018 through December 2018. Using nasopharyngeal and perirectal swabs, we evaluated the presence of XDR-AB colonization. Participants were followed up for 6 months. The primary endpoints were 28-day and 6-month mortality after ICU admission. The overall survival rate was estimated by the Kaplan-Meier method. We identified risk factors associated with 28-day and 6-month mortality using the logistic regression model and a time-dependent Cox regression model, respectively.

Results: Out of 431 patients, 77 were colonized with XDR-AB. Based on the Kaplan-Meier curve results, the overall survival before 28 days did not differ by colonization status; however, a significantly lower overall survival rate was obtained at 6 months in colonized patients. Univariate and multivariate analysis results confirmed that XDR-AB colonization was not associated with 28-day mortality, but was an independent risk factor of lower overall survival at 6 months $(\mathrm{HR}=1.749,95 \% \mathrm{Cl}=1.174-2.608)$.

Conclusions: XDR-AB colonization has no effect on short-term overall survival, but is associated with lower long-term overall survival in critically ill patients.

\footnotetext{
Keywords: Acinetobacter baumannii, extensively drug resistant, intensive care unit, colonization, infection
} 


\section{INTRODUCTION}

Acinetobacter baumannii is one of the most important opportunistic pathogens in intensive care units (ICUs) and a major cause of nosocomial infections such as hospital-acquired pneumonia, peritonitis, and bacteremia (1-3). Characterized by its environmental resilience, communicability, and wide range of drug-resistance determinants, the infection and dissemination of $A$. baumannii in critical patients can pose a heavy burden on healthcare institutions (4-6).

Increased antimicrobial resistance of $A$. baumannii has become a worldwide challenge in the care of hospitalized patients. Extensively drug-resistant A. baumannii (XDR-AB) refers to strains that lack susceptibility to almost all antimicrobial agents except for one or two (e.g., polymyxin and tigecycline) (7). Previous studies have shown that $64.6 \%$ A. baumannii isolates are XDR or PDR in European countries (8), and the XDR prevalence is $73.1-80.6 \%$ in western Asian and South American countries $(9,10)$. In China, a national investigation program on microbial resistance reported that in 2004-2014, the prevalence of XDR$\mathrm{AB}$ strains increased from 11.1 to $60.4 \%$ (11). Due to the limited availability of efficient treatments, XDR-AB infections contribute to extended hospitalizations and increased mortality rates (12, 13), which can reach $70 \%$ in critical patients with bacteremia (12).

Apart from symptomatic infections, A. baumannii colonizes hospitals and is commonly isolated from inpatient sites (14, 15). Due to the extraordinary environmental persistence and prevalence of extensive drug resistance, it is difficult to eradicate XDR-AB strains. Colonization may precede infections in severely ill patients and lead to poor prognoses. Carbapenemaseproducing Enterobacteriaceae colonization has been proven to be associated with increased risk of mortality in ICU patients in a previous study (16). However, the effect of XDR-AB colonization in critically ill patients, especially its effect on longterm prognosis, is not clear. The aim of this study was to evaluate the impact of XDR-AB colonization on both the short-term and long-term mortality of critical patients.

\section{METHODS \\ Study Design}

We conducted a prospective observational study in two mixed adult ICUs of Qilu Hospital of Shandong University from March 2018 through December 2018. Participants were followed up for 6 months after ICU admission. The study was approved by the Ethics Committee at Qilu Hospital. The need for informed consent was waived off.

Abbreviations: APACHE, Acute Physiology and Chronic Health Evaluation; CCI, Charlson Comorbidity Index; CI, confidence interval; CLSI, American Clinical and Laboratory Standards Institute; COPD, chronic obstructive pulmonary disease; CRAB, carbapenem-resistant Acinetobacter baumannii; EUCIC, European Committee on Infection Control; HR, hazard ratio; ICU, intensive care unit; IQR, interquartile range; $\mathrm{MDR}-\mathrm{AB}$, multiple drug-resistant Acinetobacter baumannii; OR, odds ratio; SD, standard deviation; WHO, World Health Organization; XDR-AB, extensively drug-resistant Acinetobacter baumannii.

\section{Participants}

Patients admitted to ICUs were considered for the study. Patients were excluded if they (1) had a length of ICU stay $<72 \mathrm{~h}$; (2) were $<18$ years of age; (3) were lost to follow-up or had insufficient information; or (4) were assumed to have colonization prior to ICU admission. We recorded essential information of the patients (e.g., age, sex, diagnosis on admission, previous medical history, and comorbidities) upon ICU admission. We calculated the Charlson Comorbidity Index (CCI) to evaluate the mortality risk associated with comorbidities $(17,18)$. We used the Acute Physiology and Chronic Health Evaluation II (APACHE II) score (19) to assess the severity of illness. The APACHE II scores were calculated within $24 \mathrm{~h}$ of ICU admission. Administration of vasoactive drugs and mechanical ventilation was documented. XDR-AB infection during admission was assessed by an independent multidisciplinary panel of experts that included one infectious disease specialist and one intensivist.

\section{Microbiology}

Nasopharyngeal and perirectal swabs were collected within 2 days after ICU admission for microbial culture and twice a week thereafter until a positive XDR-AB culture was obtained. If the patient stayed for more than 3 weeks, culture frequency was reduced to once a week. All samples were analyzed at the hospital's laboratories according to standard operating procedures. We performed strain identification and drug sensitivity testing with the VITEK2 COMPACT automatic microbial analysis system (bioMérieux, Marcy-l'Étoile, France). The susceptibility test breakpoints were based on the standards established by the American Clinical and Laboratory Standards Institute (CLSI) (20). A. baumannii (genospecies 2) was identified based on the 16S-23S ribosomal RNA gene intergenic spacer region (21).

\section{Definitions}

We defined XDR-AB according to the guidelines of the US Centers for Disease Control and Prevention and World Health Organization (WHO) (7). Colonization with XDR-AB was defined as a positive XDR-AB culture during the ICU stay. If the first culture of $\mathrm{XDR}-\mathrm{AB}$ is positive, it is assumed that the colonization occurred before ICU admission. Colonization is assumed if a negative culture is obtained before a positive one (16). The variables associated with mortality included age, sex, seasons, comorbidities and Charlson Index, admission source, primary reasons for ICU admission, intensity of care (renal replacement therapy, invasive ventilation, and vasopressor treatment), and APACHE II score.

\section{Statistical Analyses}

We used SPSS 16.0 (SPSS Inc, IL, USA) and R software v3.0 for data analysis. The primary endpoint was overall survival at 28 days and 6 months after ICU admission. Quantitative data with normal distribution were expressed as mean \pm standard deviations and compared using the Student $t$-test. Non-normally distributed data were analyzed using Wilcoxon's rank-sum test. Categorical variables were expressed as frequency (percentage) 
TABLE 1 | Descriptive results of potential risk factors and outcomes.

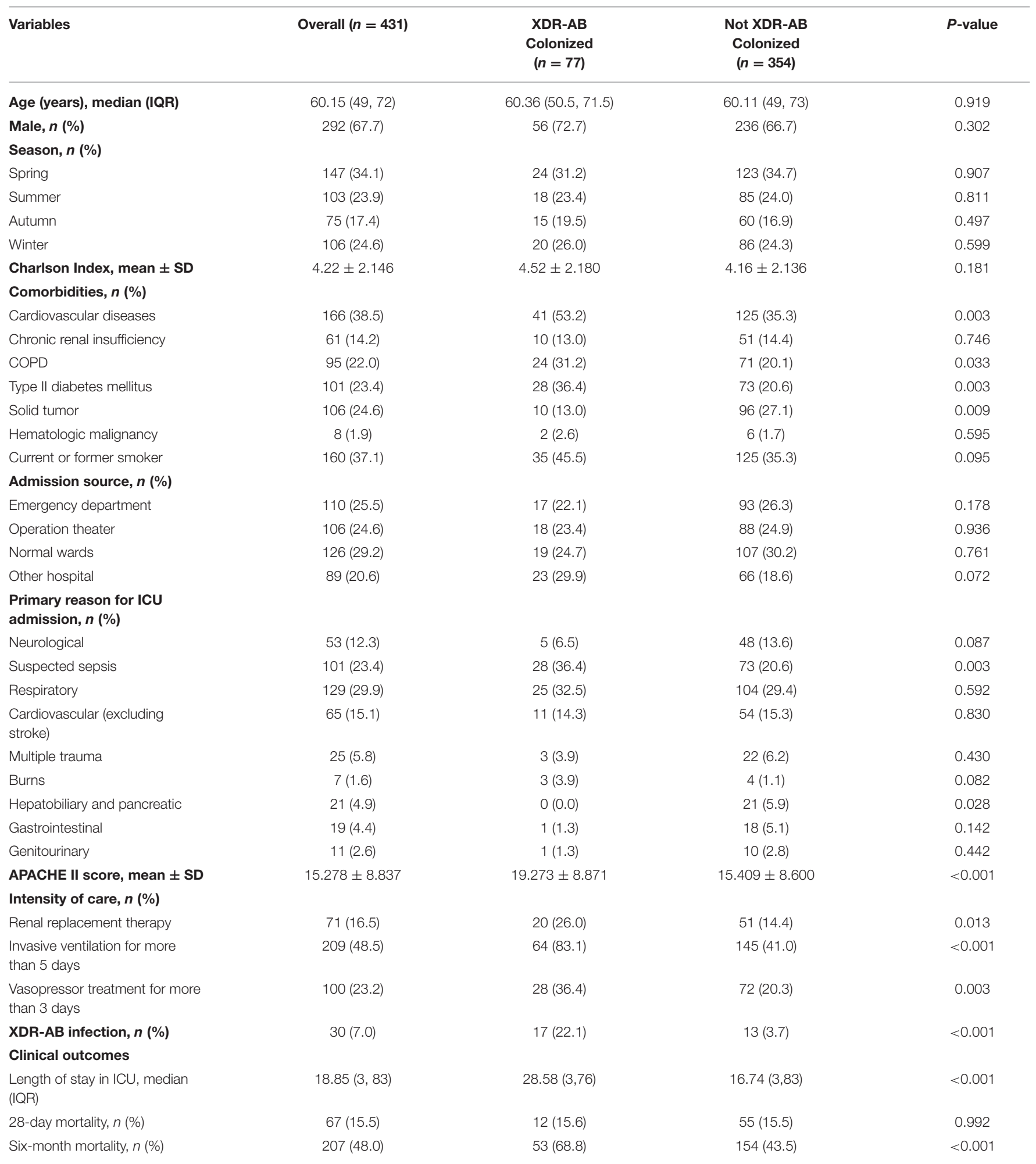

Data are number (\%), median (IQR), or mean \pm SD. XDR-AB, extensively drug-resistant Acinetobacter baumannii; COPD, chronic obstructive pulmonary disease; ICU, intensive care unit; APACHE, Acute Physiology and Chronic Health Evaluation; IQR, interquartile range; SD, standard deviation. 


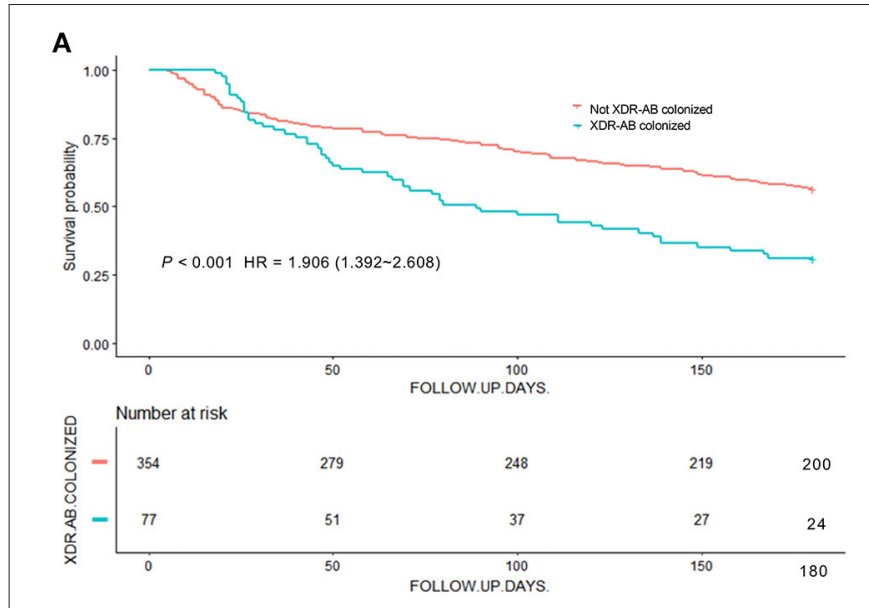

B

C

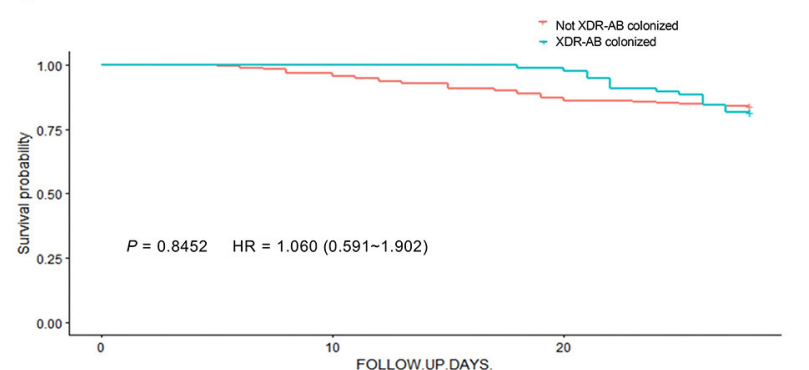

D
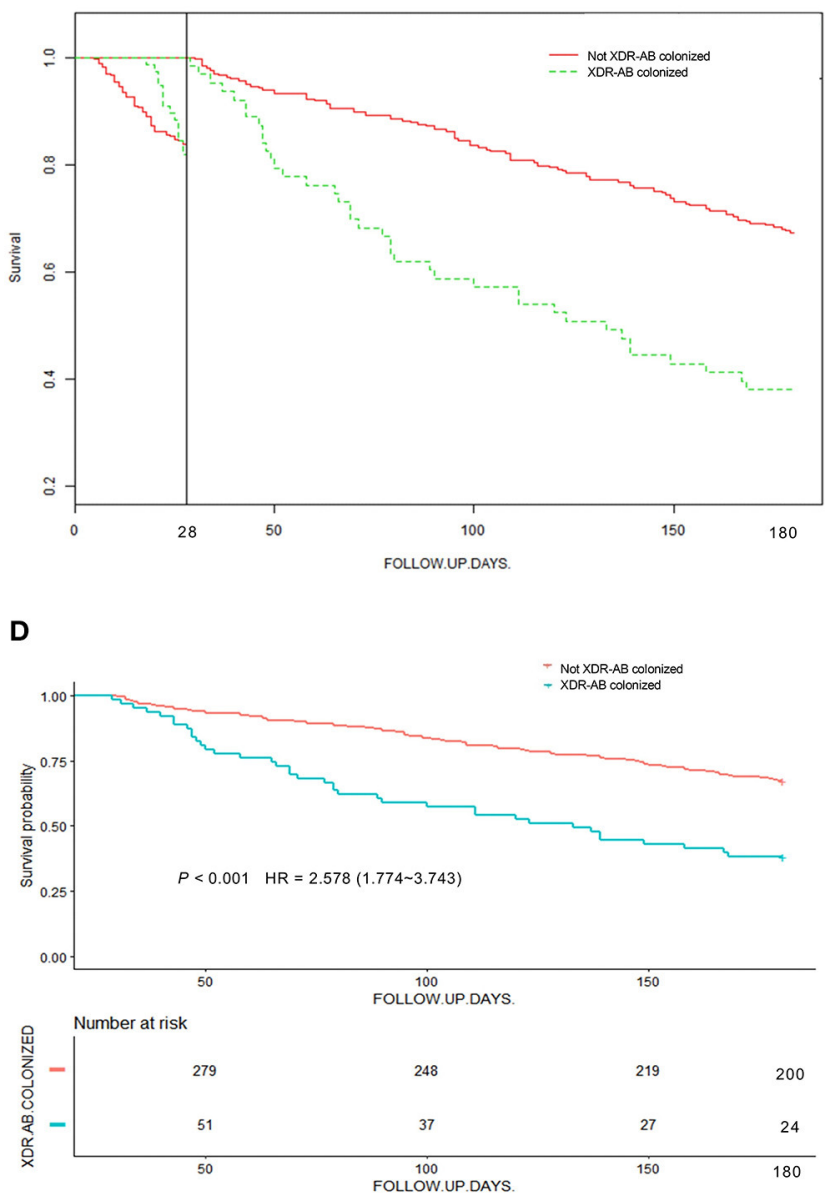

FIGURE 1 | Kaplan-Meier survival analysis stratified by XDR-AB colonization and no colonization. Survival of patients was followed for 6 months. (A) Kaplan-Meier analysis of survival in colonized and non-colonized patients. (B) Survival curves before and after 28 days. (C,D) Landmark analysis discriminating between events occurring before and after 28 days of follow-up.

and compared using the Chi-square or Fisher's exact test (twotailed). Overall survival was estimated by the Kaplan-Meier method, and the difference between Kaplan-Meier survival curves was evaluated using the log-rank test. A logistic regression model was used to explore the risk factors associated with XDR-AB colonization and 28-day mortality. Variables identified in univariate analysis with $P$-values $<0.1$ were included in the multivariate model. The Cox non-proportional hazards survival regression model was used for analyzing variables associated with mortality at 6 months. Potential time-dependent variables were tested using the proportional hazards assumption (Supplementary Table 1). XDR-AB colonization was treated as a time-dependent variable in the Cox regression model. Variables identified in univariate analysis with $P$-values $<0.1$ were included in the multivariate Cox regression models. $P$ $<0.05$ was considered statistically significant according to multivariate analysis. We performed subgroup analysis to assess the consistency of the colonization effect on survival according to the baseline variables. The Cox proportional hazards model with
Efron's method of handling ties was used to assess the magnitude of differences in XDR-AB colonization between patients with different baseline characteristics.

\section{RESULTS}

\section{Characteristics of Participants}

Out of the initial population, 237 patients were excluded, including 191 patients with expected length of stay less than 3 days, 34 patients with no culture or culture results, 6 patients lost to follow-up or with insufficient data, and 6 patients in whom the colonization was assumed to have occurred before ICU admission. A total of 431 patients were finally included in the analyses. Out of these patients, 77 (17.87\%) had XDRAB colonization. Table 1 presents the baseline characteristics and outcomes of the participants. The participants had a median age of 60 years and were mostly male (68\%). The 
TABLE 2 | Univariate analysis of factors associated with 28-day mortality.

\begin{tabular}{|c|c|c|c|c|}
\hline Variables & $\begin{array}{l}\text { Deaths at day } 28 \\
\qquad(n=67)\end{array}$ & $\begin{array}{l}\text { Survivals at day } \\
\qquad 28(n=364)\end{array}$ & $P$-value & OR $(95 \% C l)$ \\
\hline Age, median (IQR) & $61.42(53,74)$ & $59.92(49,72)$ & 0.509 & 1.005 (0.990-1.021) \\
\hline Male, $n(\%)$ & $42(62.7)$ & $250(68.7)$ & 0.336 & $1.305(0.759-2.245)$ \\
\hline \multicolumn{5}{|l|}{ Season, $n$ (\%) } \\
\hline Spring & 25 (37.3) & $122(33.5)$ & 0.547 & $1.181(0.687-2.028)$ \\
\hline Summer & $11(16.4)$ & $92(25.3)$ & 0.122 & $0.581(0.292-1.156)$ \\
\hline Autumn & $8(11.9)$ & $67(18.4)$ & 0.203 & $0.601(0.274-1.317)$ \\
\hline Winter & 23 (34.3) & $83(22.8)$ & 0.046 & $1.770(1.010-3.100)$ \\
\hline Charlson Index, mean \pm SD & $4.640 \pm 1.747$ & $4.150 \pm 2.205$ & 0.084 & $1.104(0.987-1.235)$ \\
\hline \multicolumn{5}{|l|}{ Comorbidities, $n$ (\%) } \\
\hline Cardiovascular diseases & $28(41.8)$ & $138(37.9)$ & 0.549 & $1.176(0.692-1.997)$ \\
\hline Chronic renal insufficiency & $13(19.4)$ & $48(13.2)$ & 0.183 & $1.585(0.805-3.120)$ \\
\hline COPD & $19(28.4)$ & $76(20.9)$ & 0.177 & $1.500(0.833-2.701)$ \\
\hline Type II diabetes mellitus & $17(25.4)$ & $84(23.1)$ & 0.684 & $1.133(0.621-2.069)$ \\
\hline Solid tumor & $17(25.4)$ & $89(24.5)$ & 0.872 & $1.051(0.577-1.914)$ \\
\hline Hematologic malignancy & $1(1.5)$ & $7(1.9)$ & 0.811 & $0.773(0.094-6.384)$ \\
\hline Current or former smoker & $20(29.9)$ & $140(38.5)$ & 0.182 & $0.681(0.387-1.197)$ \\
\hline \multicolumn{5}{|l|}{ Admission source, $n(\%)$} \\
\hline Emergency department & $21(31.3)$ & $89(24.5)$ & 0.236 & $1.411(0.799-2.491)$ \\
\hline Operation theater & $14(20.9)$ & $92(25.3)$ & 0.445 & $0.781(0.414-1.473)$ \\
\hline Normal wards & $21(31.3)$ & $105(28.8)$ & 0.680 & $1.126(0.641-1.979)$ \\
\hline Other hospital & $11(16.4)$ & $78(21.4)$ & 0.353 & $0.720(0.360-1.441)$ \\
\hline \multicolumn{5}{|l|}{ Primary reason for ICU admission, $n(\%)$} \\
\hline Neurological & $7(10.4)$ & $46(12.6)$ & 0.617 & $0.807(0.348-1.871)$ \\
\hline Suspected sepsis & $14(20.9)$ & $87(23.9)$ & 0.594 & $0.841(0.445-1.589)$ \\
\hline Respiratory & $20(29.9)$ & $109(29.9)$ & 0.988 & $0.996(0.563-1.759)$ \\
\hline Cardiovascular(excluding stroke) & $16(23.9)$ & $49(13.5)$ & 0.031 & $2.017(1.066-3.814)$ \\
\hline Multiple trauma & $2(3.0)$ & $23(6.3)$ & 0.295 & $0.456(0.105-1.982)$ \\
\hline Burns & $1(1.5)$ & $6(1.6)$ & 0.926 & $0.904(0.107-7.632)$ \\
\hline Hepatobiliary and pancreatic & $2(3.0)$ & $19(5.2)$ & 0.441 & $0.559(0.127-2.457)$ \\
\hline Gastrointestinal & $2(3.0)$ & $17(4.7)$ & 0.540 & $0.628(0.142-2.784)$ \\
\hline Genitourinary & $3(4.5)$ & $8(2.2)$ & 0.287 & $2.086(0.539-8.073)$ \\
\hline XDR-AB colonization, $n$ (\%) & $12(17.9)$ & $65(17.9)$ & 0.992 & $1.004(0.509-1.980)$ \\
\hline XDR-AB infection, $n$ (\%) & $7(10.4)$ & $23(6.3)$ & 0.227 & $1.730(0.711-4.210)$ \\
\hline \multicolumn{5}{|l|}{ Acuity score on admission } \\
\hline APACHE II score, mean \pm SD & $22.475 \pm 6.647$ & $13.953 \pm 8.554$ & $<0.001$ & $1.114(1.078-1.150)$ \\
\hline \multicolumn{5}{|l|}{ Intensity of care, $n$ (\%) } \\
\hline Invasive ventilation for more than 5 days & $39(58.2)$ & $170(46.7)$ & 0.085 & $1.589(0.938-2.693)$ \\
\hline Renal replacement therapy & $16(23.9)$ & $55(15.1)$ & 0.078 & $1.763(0.938-3.311)$ \\
\hline Vasopressor treatment for more than 3 days & $67(100)$ & $33(9.1)$ & 0.992 & $3.279(0.978-7.923)$ \\
\hline
\end{tabular}

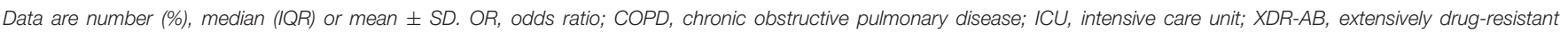
Acinetobacter baumannii; APACHE, Acute Physiology and Chronic Health Evaluation; IQR, interquartile range; SD, standard deviation.

overall 28-day and 6-month mortality rates were 15.5 and $48.0 \%$, respectively.

Patients who tested positive for XDR-AB were more likely to have cardiovascular diseases, type II diabetes mellitus, solid tumors, suspected sepsis, supportive treatment (e.g., renal replacement therapy, longer invasive ventilation, and vasopressor treatment), and $\mathrm{XDR}-\mathrm{AB}$ infections. Compared to non-colonized patients, XDR-AB colonized patients had higher APACHE II scores and 6-month mortality rates (68.8 vs. $43.5 \% ; P<0.001)$ and longer ICU days. However, 28day mortality did not differ between colonized and noncolonized patients (15.6 vs. $15.5 \%$, respectively; $P=0.992$; Table 1). Multivariate logistic analysis indicated that suspected sepsis, extended invasive ventilation, and length of stay 
TABLE 3 | Multivariate Logistic regression of Factors Associated with 28-day mortality.

\begin{tabular}{lcc}
\hline Variables & OR $(\mathbf{9 5 \%} \mathbf{C l})$ & $\boldsymbol{P}$-value \\
\hline XDR-AB colonization & $0.517(0.237-1.128)$ & 0.098 \\
APACHE II score & $1.114(1.076-1.154)$ & $<0.001$ \\
Charlson Index & $1.086(0.951-1.239)$ & 0.222 \\
Cardiovascular (excluding stroke) & $1.097(0.519-2.318)$ & 0.809 \\
Invasive ventilation for more than 5 days & $1.295(0.718-2.337)$ & 0.390 \\
Renal replacement therapy & $1.032(0.500-2.132)$ & 0.932 \\
\hline
\end{tabular}

$O R$, odds ratio; $C l$, confidence interval; $X D R-A B$, extensively drug-resistant Acinetobacter baumannii; APACHE, Acute Physiology and Chronic Health Evaluation.

in the ICU were risk factors for XDR-AB colonization (Supplementary Table 2).

\section{Kaplan-Meier Analysis of Overall Survival With Colonization Status}

Figure 1 shows that the 6-month overall survival rate was higher for non-colonized patients than for XDR-AB-colonized patients $(P<0.001)$. In the analysis of events occurring before and after the 28-day follow-up, we observed that the overall survival rate before 28 days did not differ based on the colonization status $(P=0.845)$. However, the 6 -month overall survival rate was significantly lower in colonized patients after 28 days $(P<0.001)$.

\section{Factors Predictive of 28-day Mortality}

The risk factors for 28-day mortality identified through univariate analysis include CCI, APACHE II score, ICU admission with cardiovascular diseases, support with renal replacement therapy, and extended invasive ventilation $(P<$ 0.100 for all, Table 2). The multivariate logistic regression model showed that only the APACHE II score was an independent risk factor for 28-day mortality $(\mathrm{OR}=1.114,95 \% \mathrm{CI}=1.076-1.154$; $P<0.001$; Table 3).

\section{Factors Predictive of 6-month Mortality}

Using univariate analysis, we identified possible risk factors for 6-month mortality (Table 4): age, Charlson Index, APACHE II score, presence of comorbidities (cardiovascular diseases, chronic renal insufficiency, COPD, type II diabetes mellitus, and solid tumors), admission to the ICU for primary causes (cardiovascular diseases and hepatobiliary/pancreatic diseases), renal replacement therapy, long-term invasive ventilation, vasopressor treatment, $\mathrm{XDR}-\mathrm{AB}$ colonization, and XDR-AB infection.

Considering that XDR-AB colonization and XDR-AB infection were correlated, two multivariate Cox regression models, one for each of the variables, were created. In multivariate Cox regression model 1, we found that XDR$\mathrm{AB}$ colonization was an independent risk factor for death at 6 months $(\mathrm{HR}=1.749,95 \% \mathrm{CI}=1.174-2.608)$. The other risk factors were age, high APACHE II scores, COPD, cardiovascular diseases as primary reasons for ICU admission, and extended vasopressor treatments (Table 5). In multivariate Cox regression model 2 (Supplementary Table 3), XDR-AB infection was not found to be an independent risk factor of death at 6 months.

The impact of colonization with XDR-AB in subgroups with different baseline characteristics are presented in Figure 2. In most subgroups, the number of survival days was lower for colonized patients than for non-colonized patients.

\section{DISCUSSION}

A. baumannii is one of the most threatening nosocomial microorganisms, characterized by its capacity to survive in various environments and to develop antibiotic resistance. Drug-resistant $A$. baumannii is prevalent in several healthcare facilities in China. The rapid growth of antibiotic resistance is a considerable economic and medical burden, and is associated with higher costs, longer hospital stays, and increased inhospital mortality $(22,23)$. Due to the increase in the occurrence of XDR and pan drug-resistant A. baumannii strains, WHO reported that there is an urgent need of novel antibiotics (24), emphasizing the importance of preventing and treating drugresistant $A$. baumannii.

This study assessed the effect of XDR-AB colonization on the prognoses of severely ill patients. We conducted the study in two mixed adult ICUs in China, where XDR-AB is the most common pathogen in nosocomial infections. The findings revealed that XDR-AB colonization had no association with the short-term (28-day) mortality of ICU patients but contributed to a 1.75 -fold increase in mortality risk at 6 months.

In our study, the overall peripheral colonization rate of $\mathrm{XDR}-\mathrm{AB}$ was $18 \%$, which is higher than that published in previous reports. An XDR-AB acquisition rate of $15.6 \%$ was observed in a Lebanese ICU (25). Further, in an American study, the colonization rate of XDR-AB among solid organ transplant patients was reported as $0.9 \%$ (26). The relatively high colonization rate in our study indicates a possible regional epidemic of XDR-AB in our ICUs.

Studies have elucidated several risk factors of XDR-AB colonization, such as previous admission to long-term healthcare facilities, invasive operations, presence of comorbidities, low socioeconomic status, and previous use of carbapenems (25, $27,28)$. In this study, critical patients who were admitted with suspected sepsis and required extended invasive ventilation and longer ICU stay were more likely to be colonized with XDR-AB. There are possible reasons for this finding. First, in septic patients, immune system disorders lead to poor defensive responses, making them more susceptible to opportunistic pathogens. Second, invasive operations, such as tracheal intubation and tracheostomy, provide opportunities for pathogen colonization through wounds and invasive devices due to impairments in skin and mucosal barriers. Finally, decolonization has not been adopted in ICUs in China; therefore, long-term hospitalizations coupled with poor baseline conditions and more intensive care might increase opportunities for colonization.

There is not much information on the impact of XDR$\mathrm{AB}$ colonization on patient prognosis. It has been wildly 
TABLE 4 | Univariate Analysis of Factors Associated with mortality in 6 months.

\begin{tabular}{|c|c|c|c|c|}
\hline Variables & $\begin{array}{c}\text { Deaths at } 6 \\
\text { months } \\
(n=207)\end{array}$ & $\begin{array}{c}\text { Survival at } 6 \\
\text { months } \\
(n=224)\end{array}$ & $P$-value & HR $(95 \% C l)$ \\
\hline Age, median (IQR) & $59.92(49,72)$ & $55.4(46,67.75)$ & $<0.001$ & $1.026(1.017-1.035)$ \\
\hline Male, $n(\%)$ & $138(66.7)$ & $154(68.8)$ & 0.464 & $1.114(0.834-1.487)$ \\
\hline \multicolumn{5}{|l|}{ Season, $n(\%)$} \\
\hline Spring & $70(33.8)$ & $77(34.4)$ & 0.879 & $0.978(0.733-1.304)$ \\
\hline Summer & $45(21.7)$ & $58(25.9)$ & 0.226 & $0.815(0.586-1.134)$ \\
\hline Autumn & $36(17.4)$ & $39(17.4)$ & 0.900 & $1.023(0.714-1.466)$ \\
\hline Winter & $56(27.1)$ & $50(222.3)$ & 0.190 & $1.227(0.903-1.668)$ \\
\hline Charlson Index, mean \pm SD & $4.630 \pm 2.063$ & $3.850 \pm 2.158$ & $<0.001$ & $1.114(1.056-1.175)$ \\
\hline \multicolumn{5}{|l|}{ Comorbidities, $\boldsymbol{n}$ (\%) } \\
\hline Cardiovascular diseases & $103(49.8)$ & $63(28.1)$ & $<0.001$ & $1.859(1.414-2.443)$ \\
\hline Chronic renal insufficiency & $46(22.2)$ & $15(6.7)$ & $<0.001$ & $2.231(1.605-3.101)$ \\
\hline COPD & $63(30.4)$ & $32(14.3)$ & $<0.001$ & $1.897(1.409-2.554)$ \\
\hline Type II diabetes mellitus & $56(27.1)$ & $45(20.1)$ & 0.093 & $1.301(0.957-1.769)$ \\
\hline Solid tumor & $41(19.8)$ & $65(29.0)$ & 0.064 & $0.724(0.514-1.019)$ \\
\hline Hematologic malignancy & $5(2.4)$ & $3(1.3)$ & 0.500 & $1.357(0.559-3.296)$ \\
\hline Current or former smoker & $73(35.3)$ & $87(38.8)$ & 0.322 & $0.866(0.651-1.152)$ \\
\hline \multicolumn{5}{|l|}{ Admission source, $n(\%)$} \\
\hline Emergency department & $56(27.1)$ & $54(24.1)$ & 0.503 & $1.111(0.817-1.509)$ \\
\hline Operation theater & $51(24.6)$ & $55(24.6)$ & 0.981 & $1.004(0.732-1.377)$ \\
\hline Normal wards & $58(28.0)$ & $68(30.4)$ & 0.719 & $0.946(0.698-1.281)$ \\
\hline Other hospital & $42(20.3)$ & $47(21.0)$ & 0.737 & $0.944(0.672-1.324)$ \\
\hline \multicolumn{5}{|l|}{ Primary reason for ICU admission, $n(\%)$} \\
\hline Neurological & $22(10.6)$ & $31(13.8)$ & 0.298 & $0.791(0.508-1.230)$ \\
\hline Suspected sepsis & $53(25.6)$ & $48(21.4)$ & 0.363 & $1.156(0.846-1.579)$ \\
\hline Respiratory & $63(30.4)$ & $66(29.5)$ & 0.871 & $1.025(0.762-1.378)$ \\
\hline Cardiovascular(excluding stroke) & 40 (19.3) & $25(11.2)$ & 0.005 & $1.640(1.161-2.318)$ \\
\hline Multiple trauma & $9(4.3)$ & $16(7.1)$ & 0.217 & $0.656(0.337-1.281)$ \\
\hline Burns & $2(1.0)$ & $5(2.2)$ & 0.341 & $0.508(0.126-2.047)$ \\
\hline Hepatobiliary and pancreatic & $4(1.9)$ & $17(7.6)$ & 0.024 & $0.321(0.119-0.864)$ \\
\hline Gastrointestinal & $7(3.4)$ & $12(5.4)$ & 0.362 & $0.704(0.332-1.497)$ \\
\hline Genitourinary & $6(2.9)$ & $5(2.2)$ & 0.770 & $1.129(0.501-2.542)$ \\
\hline XDR-AB colonization, $n$ (\%) & $53(25.6)$ & $24(10.7)$ & $<0.001$ & $1.898(1.387-2.598)$ \\
\hline XDR-AB infection, $\boldsymbol{n}$ (\%) & $20(9.7)$ & $10(4.5)$ & 0.034 & $1.649(1.039-2.616)$ \\
\hline \multicolumn{5}{|l|}{ Acuity score on admission } \\
\hline APACHE II score, mean \pm SD & $21.936 \pm 7.406$ & $9.125 \pm 4.556$ & $<0.001$ & $1.107(1.094-1.121)$ \\
\hline \multicolumn{5}{|l|}{ Intensity of care, $\boldsymbol{n}(\%)$} \\
\hline Invasive ventilation for more than 5 days & $118(57.0)$ & $91(40.6)$ & $<0.001$ & $1.641(1.246-2.161)$ \\
\hline Renal replacement therapy & $44(21.3)$ & $27(12.1)$ & 0.005 & $1.616(1.158-2.255)$ \\
\hline Vasopressor treatment for more than 3 days & $91(44.0)$ & $9(4.0)$ & $<0.001$ & 7.849 (5.913-10.419) \\
\hline
\end{tabular}

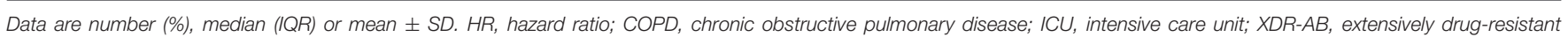
Acinetobacter baumannii; APACHE, Acute Physiology and Chronic Health Evaluation; IQR, interquartile range; SD, standard deviation.

accepted that $A$. baumannii infections can lead to higher death rates. Among critically ill patients, the estimated increase in the in-hospital mortality rate due to A. baumannii infection ranges between 7.8 and $23 \%$, and the attributable ICU mortality ranges from 10 to $43 \%$ (29). High mortality rates have been reported in patients infected with drug-resistant A. baumannii $(30,31)$. However, only a few studies have elucidated the association between mortality and colonization of drug-resistant strains. In one such cohort study conducted by Dautzenberg et al., it was confirmed that there is a 1.79 times higher risk of death in ICU patients colonized with carbapenemase-producing Enterobacteriaceae than in ICU patients who are not colonized (16). Currently, the association between A. baumannii colonization and mortality is still under debate. It has been reported that colonization with multiple drug-resistant A. baumannii (MDR-AB) strains upon ICU 
TABLE 5 | Multivariate Cox regression model for Factors Associated with mortality in 6-month (XDR-AB colonization included).

\begin{tabular}{|c|c|c|}
\hline Variables & HR $(95 \% \mathrm{Cl})$ & $P$-value \\
\hline XDR-AB colonization & $1.749(1.174-2.608)$ & 0.006 \\
\hline Age & 1.019 (1.007-1.032) & 0.002 \\
\hline APACHE II Score & $1.127(1.105-1.149)$ & $<0.001$ \\
\hline Charlson Index & $0.978(0.892-1.071)$ & 0.629 \\
\hline Presence of cardiovascular diseases & $1.013(0.712-1.442)$ & 0.942 \\
\hline Presence of chronic renal insufficiency & $1.126(0.755-1.677)$ & 0.561 \\
\hline Presence of COPD & $1.545(1.050-2.274)$ & 0.027 \\
\hline Presence of type II diabetes mellitus & $0.845(0.595-1.199)$ & 0.345 \\
\hline Presence of solid tumor & $0.857(0.545-1.347)$ & 0.503 \\
\hline Presence of hematologic malignancy & $0.998(0.365-2.728)$ & 0.997 \\
\hline Neurological diseases as primary reason for ICU admission & $0.299(0.046-1.934)$ & 0.205 \\
\hline Suspected sepsis as primary reason for ICU admission & $0.258(0.041-1.647)$ & 0.152 \\
\hline Respiratory diseases as primary reason for ICU admission & $0.262(0.041-1.668)$ & 0.156 \\
\hline Cardiovascular (excluding stroke) diseases as primary reason for ICU admission & $3.855(1.047-14.902)$ & 0.045 \\
\hline Multiple trauma as primary reason for ICU admission & $0.262(0.037-1.865)$ & 0.181 \\
\hline Burns as primary reason for ICU admission & $0.217(0.022-2.172)$ & 0.193 \\
\hline Hepatobiliary and pancreatic diseases as primary reason for ICU admission & $0.058(0.007-1.045)$ & 0.108 \\
\hline Gastrointestinal diseases as primary reason for ICU admission & $0.179(0.025-1.304)$ & 0.090 \\
\hline Genitourinary diseases as primary reason for ICU admission & $0.286(0.037-2.191)$ & 0.228 \\
\hline Invasive ventilation for more than 5 days & $0.858(0.624-1.178)$ & 0.343 \\
\hline Renal replacement therapy & $0.962(0.642-1.443)$ & 0.852 \\
\hline Vasopressor treatment for more than 3 days & $8.295(5.710-12.051)$ & $<0.001$ \\
\hline
\end{tabular}

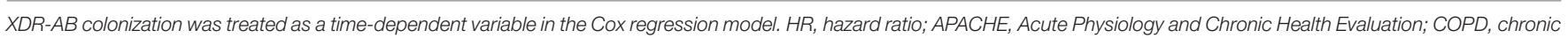
obstructive pulmonary disease; ICU, intensive care unit; XDR-AB, extensively drug-resistant Acinetobacter baumannii.

admission is related with a 1.4 -fold increase in in-hospital death rate (32). Additionally, several studies have concluded that colonization and infection with $A$. baumannii is an independent risk factor of mortality (27, 33-35), without distinguishing between colonization and infection. In this study, we identified colonization using nasopharyngeal and perirectal samplings, which are generally used to identify colonization $(16,36,37)$. As far as we know, this is the first research to assess the effect of XDR-AB colonization on long-term mortality in critical patients. The results revealed that XDR$\mathrm{AB}$ colonization has no impact on the 28 -day prognosis based on the multivariate analysis findings, but it was associated with higher mortality rates at 6 months. Thus, in our study, patients with XDR-AB colonization had a 1.75 times higher risk of death at 6 months. After adjusting for severity of illness, previous medical history, intensity of care, and other factors identified in univariate analysis, XDR-AB colonization proved to be an independent risk factor of poor longterm prognoses.

The mechanisms of bacterial colonization may hold the answer to the increased mortality observed in patients with $\mathrm{XDR}-\mathrm{AB}$ colonization. In general, bacterial colonization in hosts typically occurs in several steps. First, colonizers enter the nasopharynx and escape from the mucus, and following this, they attach themselves and adhere to epithelial cells. At the colonization loci, pathogens acquire the nutrition required to grow and to proliferate via various pathways of carbohydrate transportation and utilization (38, 39), and eventually evade host immune responses and achieve persistence. In the case of $A$. baumannii, colonization starts with pili-mediated twitching, and adherence is assisted by cell surface hydrophobicity and biofilm-associated proteins $(38,40)$. Insufficient nutrient availability in the upper respiratory tract promotes the transformation from motion to colonization (41). Additionally, persistent invasion of the host immune system is facilitated by capsules, immunoglobulin-targeted proteases, and the biofilm (42-44). The colonization is also dependent on the establishment of balance between hosts and pathogens: Immunocompetent hosts tend to coexist with the microorganisms, while infection occurs in deteriorated immune systems.

The association between $A$. baumannii colonization and mortality could be explained by several mechanisms. For example, virulence factors for A. baumannii, particularly porins, can cause cytotoxicity and induce immune responses. Porins, including OmpA, Omp34, and carO, exist on the outer membranes of $A$. baumannii. They have been found to be correlated with antimicrobial resistance, inflammatory responses, and cell death $(45,46)$. OmpA is also found in secreted membrane vesicles, and increase in the density of OmpA has been proven to be associated with high mortality (47). Additionally, spread of colonized pathogens into the lower respiratory tract, or other sterile locations, can lead to increased risk of diffuse infections (48). Moreover, interactions between colonizers can 


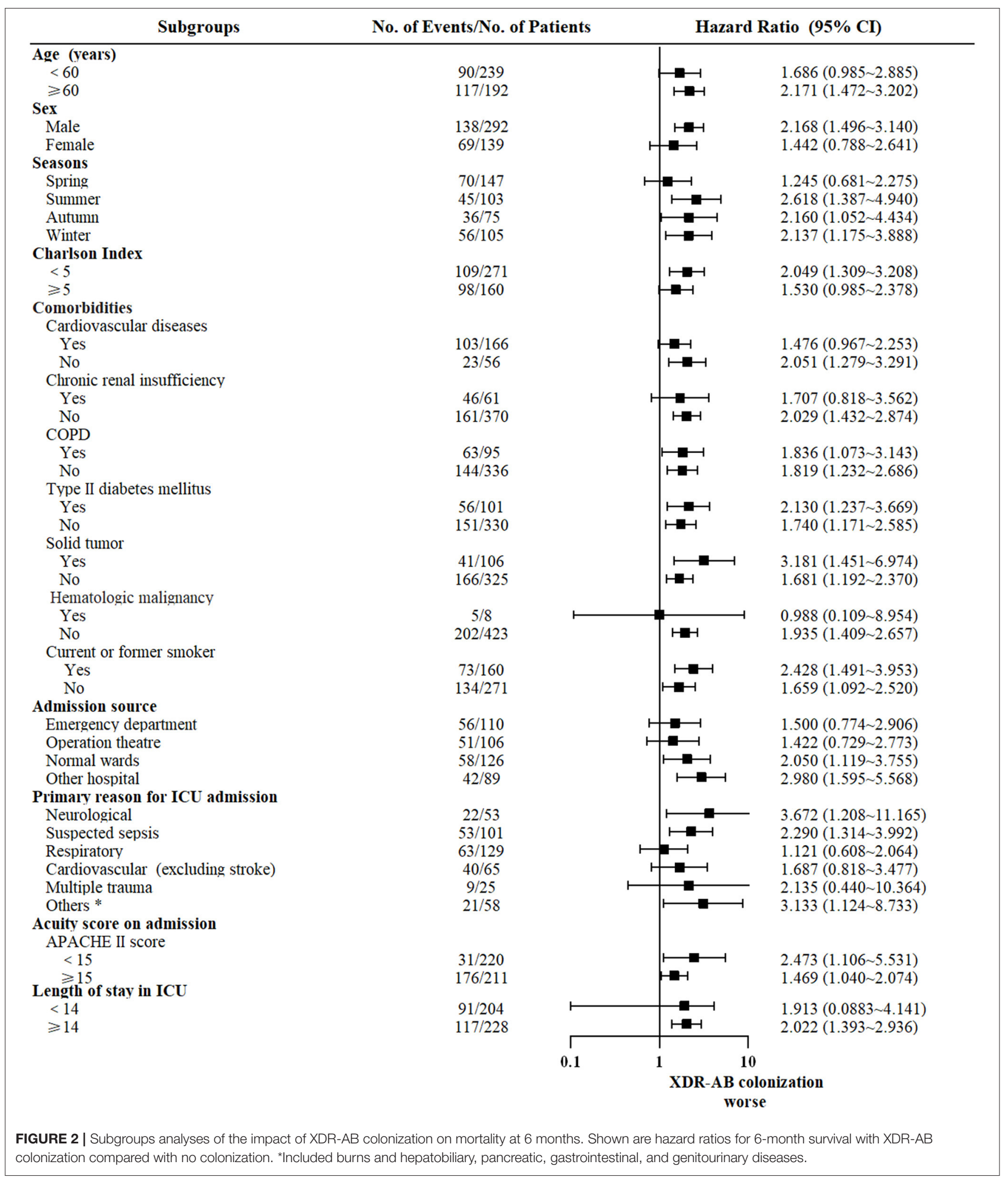

result in coinfections or induction of antibiotic resistance even without antibiotic exposure. In fact, this appears to be a prominent mechanism in bacteria with extensive drug resistance.
Increase in mortality caused by XDR-AB colonization indicates the need for essential surveillance during the early stage and efficient measures of decolonization. 
A meta-analysis reported that decolonization reduces infection caused by multidrug-resistant gram-negative bacteria when combined with standard care, especially in Europe, where decolonization has been widely applied as an infection prevention and control strategy (49). However, considering that there is a lack of effective antibiotics, it is challenging to eliminate XDR-AB. It has been reported that daily whole-body bathing with chlorhexidine was efficient in eliminating MDR-AB from the skin (50) and was helpful in reducing bloodstream infections in patients colonized with XDR gram-negative bacilli (51). Thus, this might be a feasible approach to decolonize XDR-AB. Decontamination of the alimentary tract with polymyxin $\mathrm{E}$ and tobramycin was effective in patients colonized with MDR-AB (37, 52-54). However, there is no clinical evidence about systematic antibiotics for the decolonization of XDR-AB. Additionally, standard nosocomial care is of vital importance, e.g., hand hygiene, exposure precautions, conventional screening, and environmental sterilization, especially in wards with highly prevalent strains (3). However, according to the guidelines established by the European Committee on Infection Control (EUCIC), there is not enough information about the decolonization of carbapenem-resistant Acinetobacter baumannii (CRAB) (55). Our data provide some evidence supporting the need for decolonization, but further interventional studies are required for strategy development and efficacy validation.

It has been acknowledged that in critical patients, colonization with gram-negative bacteria contributes to more nosocomial infections (56). The risk of developing subsequent $A$. baumannii infections is 8.4 times higher in patients colonized with $\mathrm{CRAB}$ (36). In this study, the incidence of XDR-AB infection is higher in colonized patients than in non-colonized ones. We also observed higher use of colistin or tigecycline after detection of XDR-AB colonization. Multivariate Cox regression analysis showed that $\mathrm{XDR}-\mathrm{AB}$ infection is not an independent risk factor for death at 6 months. This implies that the increase in the 6-month mortality of colonized patients was not caused by subsequent $\mathrm{XDR}-\mathrm{AB}$ infection during admission.

Subgroup analyses of 6-month mortality rates were performed in patients with different characteristics, and the results were consistent. Patients colonized with XDR-AB had the worse prognosis (with $\mathrm{HR}>1$ ) in all the groups; however, some of the subgroups did not show statistically significant differences. Colonization with $\mathrm{XDR}-\mathrm{AB}$ was recognized as a risk factor of 6-month mortality regardless of age, admission season, comorbidity, and APACHE II scores upon admission. In addition, XDR-AB colonization was worse in ICU patients who were hospitalized for more than 14 days $(\mathrm{HR}=2.022,95 \% \mathrm{CI}$ $=1.393-2.936)$. This indicates the harmful effect of XDR-AB colonization on critical patients with prolonged ICU stay.

Our study had some limitations. First, there is no information on the effect of colonization with drug-sensitive A. baumannii or MDR-AB on patient prognosis. Approximately $95 \%$ of the $A$. baumannii strains isolated were XDR strains, and sensitive and multidrug-resistant strains were rare. Second, we assumed in colonized patients, the XDR-AB colonization persisted at discharge, because decolonization measures were not performed. Third, information about XDR-AB infection from the patients' discharge to 6 months after discharge could not be obtained; therefore, its influence on 6-month mortality may have been underestimated. Fourth, we did not determine cause-specific survival as we were unable to obtain follow-up data for all the patients. Patients were readmitted to other hospitals that used different information systems, so it was difficult to obtain their medical records after discharge.

\section{CONCLUSIONS}

Our study provided some evidence for the impact of XDR-AB colonization on the prognosis of critically ill patients. XDR-AB colonization had no effect on the short-term mortality of ICU patients; however, it increased the 6-month mortality rates by 1.75 -fold. In the future, efficient prevention and control methods must be investigated for the management of nosocomial drugresistant $A$. baumannii.

\section{DATA AVAILABILITY STATEMENT}

The raw data supporting the conclusions of this article will be made available by the authors, without undue reservation.

\section{ETHICS STATEMENT}

The studies involving human participants were reviewed and approved by the Ethics Committee of Qilu Hospital, Shandong University. Written informed consent for participation was not required for this study in accordance with the national legislation and the institutional requirements.

\section{AUTHOR CONTRIBUTIONS}

YZ contributed to data interpretation and manuscript preparing. NX contributed to information collection and data analysis. JP contributed to manuscript preparing. $\mathrm{HH}$ and $\mathrm{HY}$ helped data interpretation. WQ, HZ, and WL helped data collection. HW and YC contributed to study design and manuscript writing. All authors approved the final manuscript and are responsible for the content.

\section{FUNDING}

This work was supported by grants from National Natural Science Foundation of China (81873927, 82072231), China Postdoctoral Science Foundation (2018M632685), Clinical Research Center of Shandong University (2020SDUCRCC013), and Shandong Key Research and Development Program (2019GSF108130).

\section{ACKNOWLEDGMENTS}

We thank Dr. Xiaorong Yang and Dr. Shaowei Sang from Clinical Epidemiology Unit, Qilu Hospital of Shandong University for 
their assistance in statistics. The former version of manuscript has been deposited on Research Square as a preprint (57), and it is available on https://www.researchsquare.com/article/ rs-225795/v1. We appreciate the efforts of the platform in academic communication.

\section{REFERENCES}

1. Falagas ME, Karveli EA, Siempos II, Vardakas KZ. Acinetobacter infections: a growing threat for critically ill patients. Epidemiol Infect. (2008) 136:1009-19. doi: 10.1017/S0950268807009478

2. Perez F, Hujer AM, Hujer KM, Decker BK, Rather PN, Bonomo RA. Global challenge of multidrug-resistant Acinetobacter baumannii. Antimicrob Agents Chemother. (2007) 51:3471-84. doi: 10.1128/AAC.01464-06

3. Chinese XDRCWG, Guan X, He L, Hu B, Hu J, Huang X, et al. Laboratory diagnosis, clinical management and infection control of the infections caused by extensively drug-resistant Gram-negative bacilli: a Chinese consensus statement. Clin Microbiol Infect. (2016) 22(Suppl. 1):S1525. doi: 10.1016/j.cmi.2015.11.004

4. Dijkshoorn L, Nemec A, Seifert H. An increasing threat in hospitals: multidrug-resistant Acinetobacter baumannii. Nat Rev Microbiol. (2007) 5:939-51. doi: 10.1038/nrmicro1789

5. Garnacho-Montero J, Dimopoulos G, Poulakou G, Akova M, Cisneros JM, De Waele J, et al. Task force on management and prevention of Acinetobacter baumannii infections in the ICU. Intensive Care Med. (2015) 41:2057-75. doi: 10.1007/s00134-015-4079-4

6. Wong D, Nielsen TB, Bonomo RA, Pantapalangkoor P, Luna B, Spellberg B. Clinical and pathophysiological overview of acinetobacter infections: a century of challenges. Clin Microbiol Rev. (2017). 30:409-47. doi: 10.1128/CMR.00058-16

7. Magiorakos AP, Srinivasan A, Carey RB, Carmeli Y, Falagas ME, Giske $\mathrm{CG}$, et al. Multidrug-resistant, extensively drug-resistant and pandrugresistant bacteria: an international expert proposal for interim standard definitions for acquired resistance. Clin Microbiol Infect. (2012) 18:268-81. doi: 10.1111/j.1469-0691.2011.03570.x

8. Nowak J, Zander E, Stefanik D, Higgins PG, Roca I, Vila J, et al. High incidence of pandrug-resistant Acinetobacter baumannii isolates collected from patients with ventilator-associated pneumonia in Greece, Italy and Spain as part of the MagicBullet clinical trial. J Antimicrob Chemother. (2017) 72:3277-82. doi: $10.1093 / \mathrm{jac} / \mathrm{dkx} 322$

9. Mirzaei B, Bazgir ZN, Goli HR, Iranpour F, Mohammadi F, Babaei R. Prevalence of multi-drug resistant (MDR) and extensively drug-resistant (XDR) phenotypes of Pseudomonas aeruginosa and Acinetobacter baumannii isolated in clinical samples from Northeast of Iran. BMC Res Notes. (2020) 13:380. doi: 10.1186/s13104-020-05224-w

10. Cerezales M, Ocampo-Sosa AA, Alvarez Montes L, Diaz Rios C, Bustamante Z, Santos J, et al. High prevalence of extensively drug-resistant Acinetobacter baumannii at a children Hospital in Bolivia. Pediatr Infect Dis J. (2018) 37:1118-23. doi: 10.1097/INF.0000000000001962

11. Hu FP, Guo Y, Zhu DM, Wang F, Jiang XF, Xu YC, et al. Resistance trends among clinical isolates in China reported from CHINET surveillance of bacterial resistance, 2005-2014. Clin Microbiol Infect. (2016) 22(Suppl. 1):S9-14. doi: 10.1016/j.cmi.2016.01.001

12. Lee HY, Chen CL, Wu SR, Huang CW, Chiu CH. Risk factors and outcome analysis of Acinetobacter baumannii complex bacteremia in critical patients. Crit Care Med. (2014) 42:1081-8. doi: 10.1097/CCM.00000000000 00125

13. Fu Q, Ye H, Liu S. Risk factors for extensive drug-resistance and mortality in geriatric inpatients with bacteremia caused by Acinetobacter baumannii. Am J Infect Control. (2015) 43:857-60. doi: 10.1016/j.ajic.2015.03.033

14. Marchaim D, Navon-Venezia S, Schwartz D, Tarabeia J, Fefer I, Schwaber MJ, et al. Surveillance cultures and duration of carriage of multidrugresistant Acinetobacter baumannii. J Clin Microbiol. (2007) 45:1551-5. doi: 10.1128/JCM.02424-06

\section{SUPPLEMENTARY MATERIAL}

The Supplementary Material for this article can be found online at: https://www.frontiersin.org/articles/10.3389/fmed. 2021.667776/full\#supplementary-material

15. Hu F, Guo Y, Yang Y, Zheng Y, Wu S, Jiang X, et al. Resistance reported from China antimicrobial surveillance network (CHINET) in (2018). Eur J Clin Microbiol Infect Dis. (2019) 38:2275-81. doi: 10.1007/s10096-019-03673-1

16. Dautzenberg MJ, Wekesa AN, Gniadkowski M, Antoniadou A, Giamarellou $\mathrm{H}$, Petrikkos GL, et al. The association between colonization with carbapenemase-producing enterobacteriaceae and overall ICU mortality: an observational cohort study. Crit Care Med. (2015) 43:1170-7. doi: 10.1097/CCM.0000000000001028

17. Charlson ME, Pompei P, Ales KL, MacKenzie CR. A new method of classifying prognostic comorbidity in longitudinal studies: development and validation. J Chronic Dis. (1987) 40:373-83. doi: 10.1016/0021-9681(87)90171-8

18. Sundararajan V, Henderson T, Perry C, Muggivan A, Quan H, Ghali WA. New ICD-10 version of the Charlson comorbidity index predicted in-hospital mortality. J Clin Epidemiol. (2004) 57:1288-94. doi: 10.1016/j.jclinepi.2004.03.012

19. Knaus WA, Draper EA, Wagner DP, Zimmerman JE. APACHE II: a severity of disease classification system. Crit Care Med. (1985) 13:818-29. doi: 10.1097/00003246-198510000-00009

20. Clinical and Laboratory Standards Institute. Performance Standards for Antimicrobial Susceptibility Testing. Wayne, PA: Clinical and Laboratory Standards Institute (2017).

21. Lin YC, Sheng WH, Chang SC, Wang JT, Chen YC, Wu RJ, et al. Application of a microsphere-based array for rapid identification of Acinetobacter spp. with distinct antimicrobial susceptibilities. J Clin Microbiol. (2008) 46:612-7. doi: 10.1128/JCM.01798-07

22. Zhen $\mathrm{X}$, Stalsby Lundborg $\mathrm{C}$, Sun $\mathrm{X}$, Zhu N, Gu S, Dong $\mathrm{H}$. Economic burden of antibiotic resistance in China: a national level estimate for inpatients. Antimicrob Resist Infect Control. (2021) 10:5. doi: 10.1186/s13756-020-00872-w

23. Zhen X, Chen Y, Hu X, Dong P, Gu S, Sheng YY, et al. The difference in medical costs between carbapenem-resistant Acinetobacter baumannii and non-resistant groups: a case study from a hospital in Zhejiang province, China. Eur J Clin Microbiol Infect Dis. (2017) 36:1989-94. doi: 10.1007/s10096-017-3088-3

24. WHO Publishes List of Bacteria for Which New Antibiotics Are Urgently Needed. Available online at: https://www.who.int/news/item/27-02-2017who-publishes-list-of-bacteria-for-which-new-antibiotics-are- urgentlyneeded

25. Moghnieh R, Siblani L, Ghadban D, El Mchad H, Zeineddine R, Abdallah D, et al. Extensively drug-resistant Acinetobacter baumannii in a Lebanese intensive care unit: risk factors for acquisition and determination of a colonization score. J Hosp Infect. (2016) 92:47-53. doi: 10.1016/j.jhin.2015.10.007

26. Shields RK, Clancy CJ, Gillis LM, Kwak EJ, Silveira FP, Massih RC, et al. Epidemiology, clinical characteristics and outcomes of extensively drugresistant Acinetobacter baumannii infections among solid organ transplant recipients. PLoS ONE. (2012) 7:e52349. doi: 10.1371/journal.pone.0052349

27. Henig O, Weber G, Hoshen MB, Paul M, German L, Neuberger A, et al. Risk factors for and impact of carbapenem-resistant Acinetobacter baumannii colonization and infection: matched case-control study. Eur J Clin Microbiol Infect Dis. (2015) 34:2063-8. doi: 10.1007/s10096-015-2452-4

28. Kim YA, Park YS, Lee SS, Son YJ, Yeon JH, Seo YH, et al. Colonization prevalence and risk factor analysis of carbapenem-resistant Acinetobacter baumannii in an intensive care unit without outbreaks. Kjhaicp. (2019) 24:81-7. doi: 10.14192/kjicp.2019.24.2.81

29. Falagas ME, Bliziotis IA, Siempos II. Attributable mortality of Acinetobacter baumannii infections in critically ill patients: a systematic review of matched cohort and case-control studies. Crit Care. (2006) 10:R48. doi: 10.1186/cc4869 
30. Karakonstantis S, Gikas A, Astrinaki E, Kritsotakis EI. Excess mortality due to pandrug-resistant Acinetobacter baumannii infections in hospitalized patients. J Hosp Infect. (2020) 106:447-53. doi: 10.1016/j.jhin.2020.09.009

31. Shi J, Sun T, Cui Y, Wang C, Wang F, Zhou Y, et al. Multidrug resistant and extensively drug resistant Acinetobacter baumannii hospital infection associated with high mortality: a retrospective study in the pediatric intensive care unit. BMC Infect Dis. (2020) 20:597. doi: 10.1186/s12879-020-05321-y

32. Blanco N, Harris AD, Rock C, Johnson JK, Pineles L, Bonomo RA, et al. Risk factors and outcomes associated with multidrug-resistant Acinetobacter baumannii upon intensive care unit admission. Antimicrob Agents Chemother. (2018) 62:e01631-17. doi: 10.1128/AAC.01631-17

33. Gkrania-Klotsas E, Hershow RC. Colonization or infection with multidrugresistant Acinetobacter baumannii may be an independent risk factor for increased mortality. Clin Infect Dis. (2006) 43:1224-5. doi: 10.1086/508360

34. Molina J, Cisneros JM, Fernandez-Cuenca F, Rodriguez-Bano J, Ribera A, Beceiro A, et al. Clinical features of infections and colonization by Acinetobacter genospecies 3. J Clin Microbiol. (2010) 48:4623-6. doi: 10.1128/JCM.01216-10

35. Ntusi NB, Badri M, Khalfey H, Whitelaw A, Oliver S, Piercy J, et al. ICU-associated Acinetobacter baumannii colonisation/infection in a high HIV-prevalence resource-poor setting. PLoS ONE. (2012) 7:e52452. doi: 10.1371/journal.pone.0052452

36. Latibeaudiere R, Rosa R, Laowansiri P, Arheart K, Namias N, MunozPrice LS. Surveillance cultures growing carbapenem-Resistant Acinetobacter baumannii predict the development of clinical infections: a retrospective cohort study. Clin Infect Dis. (2015) 60:415-22. doi: 10.1093/cid/ciu847

37. Agustí C, Pujol M, Argerich MJ, Ayats J, Badía M, Domínguez MA, et al. Short-term effect of the application of selective decontamination of the digestive tract on different body site reservoir ICU patients colonized by multi-resistant Acinetobacter baumannii. J Antimicrob Chemother. (2002) 49:205-8. doi: 10.1093/jac/49.1.205

38. Pezzulo AA, Gutierrez J, Duschner KS, McConnell KS, Taft PJ, Ernst SE, et al. Glucose depletion in the airway surface liquid is essential for sterility of the airways. PLoS ONE. (2011) 6:e16166. doi: 10.1371/journal.pone.0016166

39. Philips BJ, Meguer JX, Redman J, Baker EH. Factors determining the appearance of glucose in upper and lower respiratory tract secretions. Intensive Care Med. (2003) 29:2204-10. doi: 10.1007/s00134-003-1961-2

40. Krasowska A, Sigler K. How microorganisms use hydrophobicity and what does this mean for human needs? Front Cell Infect Microbiol. (2014) 4:112. doi: $10.3389 /$ fcimb. 2014.00112

41. Eijkelkamp BA, Stroeher UH, Hassan KA, Papadimitrious MS, Paulsen IT, Brown MH. Adherence and motility characteristics of clinical Acinetobacter baumannii isolates. FEMS Microbiol Lett. (2011). 323:44-51. doi: 10.1111/j.1574-6968.2011.02362.x

42. Plaut AG. The IgA1 proteases of pathogenic bacteria. Annu Rev Microbiol. (1983) 37:603-22.

43. Ketter PM, Yu JJ, Guentzel MN, May HC, Gupta R, Eppinger M, et al. Acinetobacter baumannii gastrointestinal colonization is facilitated by secretory IgA which is reductively dissociated by bacterial thioredoxin A. mBio. (2018) 9:e01298-18. doi: 10.1128/mBio.01298-18

44. Hoyle BD, Costerton JW. Bacterial resistance to antibiotics: the role of biofilms. Prog Drug Res. (1991) 37:91-105.

45. Kwon HI, Kim S, Oh MH, Shin M, Lee JC. Distinct role of outer membrane protein $\mathrm{A}$ in the intrinsic resistance of Acinetobacter baumannii and Acinetobacter nosocomialis. Infect Genet Evol. (2019) 67:33-7. doi: 10.1016/j.meegid.2018.10.022

46. Gaddy JA, Arivett BA, McConnell MJ, Lopez-Rojas R, Pachon J, Actis LA. Role of acinetobactin-mediated iron acquisition functions in the interaction of Ac6inetobacter baumannii strain ATCC $19606 \mathrm{~T}$ with human lung epithelial cells, Galleria mellonella caterpillars, and mice. Infect Immun. (2012) 80:101524. doi: 10.1128/IAI.06279-11

47. Jin JS, Kwon SO, Moon DC, Gurung M, Lee JH, Kim SI, et al. Acinetobacter baumannii secretes cytotoxic outer membrane protein A via outer membrane vesicles. PLoS ONE. (2011) 6:e17027. doi: 10.1371/journal.pone.0017027

48. Albrich WC, Madhi SA, Adrian PV, van Niekerk N, Mareletsi T, Cutland $\mathrm{C}$, et al. Use of a rapid test of pneumococcal colonization density to diagnose pneumococcal pneumonia. Clin Infect Dis. (2012) 54:601-9. doi: $10.1093 / \mathrm{cid} / \mathrm{cir} 859$

49. Teerawattanapong N, Kengkla K, Dilokthornsakul P, Saokaew S, Apisarnthanarak A, Chaiyakunapruk N. Prevention and control of multidrugresistant gram-negative bacteria in adult intensive care units: a systematic review and network meta-analysis. Clin Infect Dis. (2017). 64:S51-60. doi: $10.1093 / \mathrm{cid} / \mathrm{cix} 112$

50. Borer A, Gilad J, Porat N, Megrelesvilli R, Saidel-Odes L, Peled N, et al. Impact of $4 \%$ chlorhexidine whole-body washing on multidrugresistant Acinetobacter baumannii skin colonisation among patients in a medical intensive care unit. J Hosp Infect. (2007) 67:149-55. doi: 10.1016/j.jhin.2007.07.023

51. Munoz-Price LS, Hota B, Stemer A, Weinstein RA. Prevention of bloodstream infections by use of daily chlorhexidine baths for patients at a longterm acute care hospital. Infect Control Hosp Epidemiol. (2009) 30:1031-5. doi: $10.1086 / 644751$

52. Timsit JF, Garrait V, Misset B, Goldstein FW, Renaud B, Carlet J. The digestive tract is a major site for Acinetobacter baumannii colonization in intensive care unit patients. J Infect Dis. (1993) 168:1336-7. doi: 10.1093/infdis/168.5.1336

53. Parra Moreno ML, Arias Rivera S, de la Cal López MA, Frutos Vivar F, Cerdá Cerdá E, García Hierro P, et al. Effect of selective digestive decontamination on the nosocomial infection and multiresistant microorganisms incidence in critically ill patients. Med Clin. (2002) 118:3614. doi: 10.1016/S0025-7753(02)72388-9

54. Corbella X, Pujol M, Ayats J, Sendra M, Ardanuy C, Domínguez MA, et al. Relevance of digestive tract colonization in the epidemiology of nosocomial infections due to multiresistant Acinetobacter baumannii. Clin Infect Dis. (1996) 23:329-34. doi: 10.1093/clinids/23.2.329

55. Tacconelli E, Mazzaferri F, de Smet AM, Bragantini D, Eggimann P, Huttner BD, et al. ESCMID-EUCIC clinical guidelines on decolonization of multidrug-resistant Gram-negative bacteria carriers. Clin Microbiol Infect. (2019) 25:807-17. doi: 10.1016/j.cmi.2019.01.005

56. Kerver AJ, Rommes JH, Mevissen-Verhage EA, Hulstaert PF, Vos A, Verhoef J, et al. Prevention of colonization and infection in critically ill patients: a prospective randomized study. Crit Care Med. (1988) 16:1087-93. doi: 10.1097/00003246-198811000-00001

57. Yue Z, Nana X, Jiaojiao P, Hui H, Hongna Y, Weidong Q, et al. Colonization with extensively drug-resistant Acinetobacter Baumannii and prognosis in critically ill patients: an observational cohort study. Research Square. (2021). Available online at: https://www.researchsquare.com/article/rs-225795/v1

Conflict of Interest: The authors declare that the research was conducted in the absence of any commercial or financial relationships that could be construed as a potential conflict of interest.

Copyright (C) 2021 Zheng, Xu, Pang, Han, Yang, Qin, Zhang, Li, Wang and Chen. This is an open-access article distributed under the terms of the Creative Commons Attribution License (CC BY). The use, distribution or reproduction in other forums is permitted, provided the original author(s) and the copyright owner(s) are credited and that the original publication in this journal is cited, in accordance with accepted academic practice. No use, distribution or reproduction is permitted which does not comply with these terms. 